\title{
Factores de riesgos en mujeres diagnosticadas con lesiones pre-malignas de cáncer cérvicouterino
}

Risk factors in women diagnosed with pre-malignant lesions of cervical cancer

\author{
Karelia Gallardo Mendoza' \\ Wilfred Cunningham Kain²
}

\section{Resumen}

Este estudio descriptivo cuali-cuantitativo de corte transversal, se realizó en la unidad de atención primaria en salud, Policlínico "Ernesto Hodgson" del municipio de Puerto Cabezas. El universo corresponde a 38 pacientes, se desarrollaron entrevistas dirigidas a las mujeres, también se consideraron los datos estadísticos y expedientes de la unidad.

Entre los resultados es que la principal lesión pre maligna de cáncer cervicouterino son las neoplasias leves, es decir NIC I con un 63\%, seguido de los NIC II con un 21\%, y atipias escamosas en las que se incluyen las infecciones por VPH con un 13\%. A pesar de los diferentes factores de riesgo se han logrado captar en lesiones tempranas, por lo que para lesiones severas NIC III se encontró un $3 \%$.

La mayoría de las pacientes eran del área urbana y de la etnia miskitu, ya que es el pueblo de predominio en la región. En relación a su estado civil, el 42\% de las pacientes tenían parejas sexuales con relación estable. Se concluyó que las mujeres en estudio presentan múltiples factores de riesgo para desarrollar lesiones pre malignas de cáncer cervicouterino, básicamente están los antecedentes sexuales, inicio de vida sexual activa a temprana edad, múltiples compañeros sexuales y el número de hijos.

Palabras clave: cáncer cérvicouterino; factores de riesgos; diagnóstico.

\section{Abstract}

This descriptive qualitative-quantitative cross-sectional study was carried out in the primary health care unit, "Ernesto Hodgson" Polyclinic in the Puerto Cabezas municipality. The universe corresponds to 38 patients, interviews were conducted to women, the statistical data and files of the unit were also considered.

Among the results is that the main pre malignant lesion of cervical cancer are mild neoplasms, i.e. CIN I with $63 \%$, followed by CIN II with $21 \%$, and squamous atypia in which HPV infections are included. $13 \%$ although different risk factors have been achieved in early lesions, so for severe CIN III lesions $3 \%$ was found.

The majority of the patients were from the urban area and from the Miskitu ethnic group, since it is the predominant town in the region. Regarding their marital status, $42 \%$ of the patients had sexual partners with a stable relationship. It was concluded that the women under study present multiple risk factors to develop pre malignant lesions of cervical cancer, basically there are the sexual background, the beginning of an active sexual life at an early age, multiple sexual partners and the number of children.

\footnotetext{
1 Doctora en Medicina Intercultural y Cirugía. Doctora de Medicina General y Cirugía del Ministerio de Salud de la Región Autónoma de la Costa Caribe Norte de Nicaragua. Correo: angel10051@Hotmail.com

2 Especialista en Ginecobstetricia. Doctor de Medicina General y Cirugía del Ministerio de Salud de la Región Autónoma de la Costa Caribe Norte de Nicaragua. Correo: wilfredck@hotmail.com
}

Recibido: 19/06/2018 - Aprobado: 25/01/2019 
Keywords: cervical cancer; risk factors; diagnosis

\section{Introducción}

El cáncer es una enfermedad totalmente prevenible y curable, a bajo costo y con un bajo riesgo, cuando se cuento con métodos para tamizaje en mujeres asintomáticas por lesiones pre-malignas, junto con un diagnóstico, tratamiento y seguimiento apropiados.

Los informes emitidos por la Organización Mundial de la Salud, refieren que el cáncer presenta una tendencia creciente dado que anualmente se producen alrededor de 10 millones de casos nuevos y se espera cerca de 15 millones para el año 2020 (OMS, 2011). El término se atribuye a un crecimiento anormal de células. Por consiguiente, las lesiones pre malignas de cáncer cérvico uterino son lesiones precursoras poco invasivas inicialmente que provocan modificaciones al tejido paulatinamente, las cuales suelen ser asintomáticas y silenciosas durante su desarrollo sin sintomatología aparente.

A nivel mundial el cáncer de cuello uterino es la segunda causa de morbilidad y mortalidad por cáncer en la mujer y representa el 15\% de todos los tipos de cáncer, el $80 \%$ se diagnostica en países en desarrollo. En América Latina y el Caribe sigue siendo un problema importante de salud pública entre las mujeres y aunque esta puede evitarse en gran medida, los esfuerzos colectivos para prevenirla no han logrado disminuir su carga en la región de las Américas.

En Nicaragua a través del Ministerio de la Salud y en coordinación con la sociedad civil organizada, se pretende sensibilizar al personal de salud, a la red comunitaria y a los actores sociales, acerca de la importancia de promover el auto-cuidado de la salud por medio de la toma del Papanicolaou (PAP) y la realización del examen clínico oportuno y periódicamente, para diagnosticar a tiempo cáncer y lesiones precursoras de este a tiempo.

Los principales factores de riesgos presentes en las mujeres de este municipio son de comportamiento sexual y falta de asistencia a atención ginecológica, en algunos casos por falta de prácticas de chequeos médicos o por la lejanía geográfica. Además, en la región la cosmovisión y cultura de las mujeres influye mucho en la atención a la cual acudirán para manejo de las patologías relacionadas al órgano reproductor femenino, por lo que en algunos casos tiende a predisponerlas.

\section{Revisión de literatura}

\section{El útero}

Según Schwartz (2008:03), el útero es un órgano muscular hueco, que tiene forma de una pera achatada de pared fibromuscular situada entre la vejiga y el recto. El tamaño del útero varía dependiendo de la edad y el número de embarazos de cada mujer. En la edad de reproducción pesa unos $50 \mathrm{~g}$ y mide aproximadamente $8.0 \times 6.0 \times 3.0 \mathrm{~cm}$.

\section{Cuello uterino}

El cuello uterino constituye el tercio inferior del útero y está compuesto de un tejido fibromuscular denso recubierto por dos tipos de epitelio mide unos $3 \mathrm{~cm}$ de longitud por $2.5 \mathrm{~cm}$ de diámetro. La parte inferior del cuello uterino (cuello uterino externo o exocervix se encuentra en el interior de la vagina y es visible con especulo; los dos tercios superiores (cuello uterino interno o endocervix) se encuentra por encima de la vagina. 
La zona de transformación es un tejido de alta actividad celular en el que asientan la mayoría de las lesiones pre invasoras, y es conveniente conocerla bien. Se trata de la porción del cérvix que originariamente tenía epitelio cilíndrico y ahora tiene epitelio escamoso.

Durante la vida reproductiva la unión cilindro escamosa emigra en dirección cefálica sobre el borde delantero de la zona de transformación y puede ser invisible a simple vista después de la menopausia. En esta zona de transformación que abarca a la unión cilindro escamoso es donde aparecen los carcinomas epidermoides o las lesiones pre cancerosas. El segmento inferior del útero o istmo es la porción situada entre el endocervix y cavidad endometrial.

\section{Cáncer}

Schwartz (2008:27), conceptualiza el término cáncer como: El crecimiento maligno y multiplicación incontrolada, autónoma y desregulada de las células cervicales. Dicho crecimiento da lugar a tumores que pueden invadir partes adyacentes y distantes del cuerpo, a la par que destruyen tejidos normales y compiten por los nutrientes y oxígeno. Se puede desarrollar a partir de cualquiera de los tejidos que componen el mismo, fundamentalmente a partir de los epitelios de revestimiento

La OPS (2002), indica que hay casi 1,4millones de casos de cáncer de cuello uterino reconocidos clínicamente. Este cálculo refleja la acumulación de los casos producidos cada año y el hecho de que pocas mujeres de los países en desarrollo reciben tratamiento.

La OMS (2000), menciona que, en la mayoría de los países, la incidencia del cáncer invasor del cuello uterino es muy baja en las mujeres menores de 25 años. La incidencia aumenta alrededor de los 35 a 40 años y alcanza un máximo en las mujeres de entre 50 y 70 años de edad. Las tasas de incidencia por edad más elevadas de lesiones pre malignas de cuello uterino se han registrado en Melanesia, África meridional, Centroamérica y América del sur

La OPS (2002), menciona que, las áreas de mayor riesgo están en América central y Suramérica, el sureste y este de África y en el Caribe, donde la incidencia promedio es superior de $4^{\circ}$ por 100.000 mujeres afectadas con lesiones por año y llega hasta un 20 o 30\% de todas las neoplasias. Las mujeres latinas de 35 a 55 años de edad padecen con mayor frecuencia cáncer de cuello uterino y lesiones precursoras de este, a pesar de las campañas de detección oportuna

\section{Factores de Riesgo}

Los estudios han encontrado varios factores que pueden aumentar el riesgo de desarrollar lesiones pre malignas y por tanto de padecer cáncer cérvico uterino. Por ejemplo, la infección con el virus del papiloma humano (VPH) es la causa principal del cáncer cervical.

La infección del VPH y otros factores pueden actuar en conjunto para aumentar aún más el riesgo: Los VPH son un grupo de virus que pueden infectar el cérvix, La infección genital con el virus del papiloma humano (VPH) es la enfermedad de transmisión sexual viral más frecuente en el mundo.

Irregularidad en hacerse pruebas de Papanicolaou: El cáncer cervical es más común en mujeres que no se hacen pruebas regulares de Papanicolaou. La prueba de Papanicolaou ayuda a que los médicos encuentren células alteradas o lesiones precursoras de cáncer.

La ausencia de tamizaje o pesquisa es una técnica utilizada para la detección de una enfermedad en etapas iníciales. Se utiliza idealmente en individuos asintomáticos, a quienes se realiza un examen o procedimiento para detectar la enfermedad incipiente. 
Sistema inmunitario debilitado (el sistema natural de defensa del cuerpo): Mujeres con estado inmunológico deficiente, como son las infectadas con el virus de la inmunodeficiencia humana (VIH) o sometidas a drogas inmunosupresoras, son más sensibles a la infección de varios tipos de VPH y al desarrollo de cáncer cervicouterino. Se ha descrito una prevalencia de infección con VPH 2 a 4 veces mayor en seropositivas a VIH en relación a las seronegativas.

Schwartz define antecedentes sexuales: Mujeres que han tenido múltiples compañeros sexuales (promiscuidad), o han tenido compañeros sexuales de alto riesgo (es decir que han tenido muchas compañeras sexuales y/o comenzaron relaciones sexuales a temprana edad). Inicio de relaciones sexuales a temprana edad: la edad del primer coito antes de los 20 y de manera marcada antes de los 18 años es uno de los cofactores importantes en la aparición del carcinoma del cuello uterino. Generalmente, el inicio temprano de las relaciones implica la aparición de múltiples compañeros sexuales, con el consiguiente riesgo dado por estos. Se ha demostrado también que en la adolescencia los tejidos cérvicos uterinos son más susceptibles a la acción de los carcinógenos, y de hecho, si existe un agente infeccioso relacionado, el tiempo de exposición a este será mucho mayor. Compañero sexual de alto riesgo: si tiene antecedentes de cáncer de pene, antecedentes de relaciones sexuales con una mujer con neoplasia cervical, visita frecuentemente a trabajadoras del sexo, nunca utiliza condón.

Es imprescindible considerar la influencia del hombre en la génesis de lesiones pre malignas de cáncer cervicouterino y sobre todo en el varón de riesgo o promiscuo que mantiene relaciones sexuales sin protección de barrera como el condón, este tiene una probabilidad más alta de desarrollar una infección de transmisión sexual (ITS) y por tanto, una contaminación en su plasma seminal, la cual tiene importante papel en la infección por el VPH.

\section{Aspecto socioeconómico}

FUNICA, (2009). En términos generales, las condiciones de pobreza se han asociado a mayor riesgo de adquirir ciertas enfermedades, entre ellas, lesiones precursoras de cáncer cervicouterino. Las mujeres con menor nivel socioeconómico están más expuestas a varios factores de riesgo descritos para el desarrollo de la enfermedad, como son, el menor acceso a controles de salud (y con ello menor tasa de tamizaje y detección precoz), menor educación y conductas sexuales de mayor riesgo. El estado nutricional e inmunológico deficiente, asociado a esta condición, también favorece la infección por VPH

Antecedentes familiares de Cacu: El cáncer de cuello uterino puede presentarse con mayor frecuencia en algunas familias. Algunos investigadores sospechan que algunos casos de esta tendencia familiar son causados por una condición hereditaria que hace que algunas mujeres sean menos capaces de luchar contra la infección con VPH que otras.

\section{Características culturales}

Avelino Cox (Año por favor), refiere que este es un factor que puede predisponer en algunos casos ya que nuestra área de estudio posee diversas culturas y cosmovisión desde las cuales el binomio salud- enfermedad se proyecta desde muchos puntos de vista, para las mujeres indígenas este tipo de enfermedades se relaciona estrechamente con la diosa del agua la sirena o liwa mairin por lo tanto debería ser tratada por sus médicos tradicionales, lo que en su mayoría crea una brecha en la captación, control, manejo y seguimiento de estas mujeres cuando no hay vínculos entre el manejo tradicional y convencional.

Este es un aspecto que desde otras literaturas internacionales no se toma en cuenta ya que no existe o se practica muy poco el manejo desde un punto intercultural de las enfermedades de los pueblos indígenas en este caso las relacionadas al órgano femenino. 


\title{
Clasificación de las lesiones pre malignas
}

\author{
Sistema de clasificación Richard (1967)
}

NIC1 (Neoplasia intracervical 1 o Grado I), es el tipo de menor riesgo, representa sólo una displasia leve o crecimiento celular anormal y es considerado una lesión escamosa intraepitelial de bajo grado. Se caracteriza por estar confinado al $1 / 3$ basal del epitelio cervical.

NIC se definió como: "un espectro de condiciones que se inicia con alteraciones epiteliales bien diferenciadas, tradicionalmente llamadas displasia mínima y que termina al convertirse en carcinoma micro invasor"

$\mathrm{NIC}_{2}$ (Grado II), es considerado una lesión escamosa intraepitelial de alto grado3 y representan una displasia moderada, confinada a los 2/3 basales del epitelio cervical.

Cáncer in situ es el término histológico que abarca el conjunto de alteraciones de la maduración y diferenciación epitelial, correspondiente al NIC II y NIC III. Constituye la verdadera lesión precursora del cáncer de cérvix, que dejado a evolución espontánea, progresaría a una lesión maligna en muchos casos.

$\mathrm{NIC}_{3}$ (Grado III): en este tipo de lesión, considerada también de alto grado, la displasia es severa y cubre más de los $2 / 3$ de todo el epitelio cervical, en algunos casos incluyendo todo el grosor del revestimiento cervical. Esta lesión es considerada como un carcinoma insitu.

\section{Tratamiento}

\section{Manejo de las lesiones pre invasoras de cuello uterino}

Lesión intraepitelial de bajo grado (LIE-BG), infección por virus del papiloma humano (VPH) y neoplasia intracervical I (NIC I): Para el tratamiento, hay que tener en cuenta que las Lesiones de Bajo Grado en un buen porcentaje (40-60\%) pueden tener regresión espontáneamente, por lo que es necesario particularizar cada caso, ver las características de la usuaria, las posibilidades de seguimiento y las características de la lesión.

Los cuatro métodos más utilizados para el tratamiento de lesión debajo grado son: Observación (seguimiento a través de citología), Crioterapia, Excisión electro quirúrgica con ASA, Vaporización láser.

La conducta cambia de acuerdo a las circunstancias y cada caso debe ser analizado individualmente, teniendo en cuenta: La edad de la usuaria, Paridad y deseos de gestaciones futuras, La localización y extensión de la lesión, El riesgo quirúrgico, la facilidad de seguimiento de la usuaria. Unas situaciones especiales representan las usuarias jóvenes o con deseo de embarazo portadoras de lesiones NIC II, pero que cumplan las siguientes características: Lesiones pequeñas, localizadas y limitadas estrictamente en exocervix. visibles en toda su extensión, sin compromiso del canal.

Se podría utilizar la crioterapia o la vaporización láser, sin embargo, debe tomarse en cuenta que idealmente debe preferirse el procedimiento con asa térmica, conos poco profundos que permiten extraer la zona de transformación y la lesión para estudio histológico que nos brinda certeza diagnóstica y terapéutica.

En las lesiones de NIC III, siempre se prefieren los métodos de escisión para obtener una muestra adicional para patología, de esta forma se confirma la extracción de la lesión completa y el estado de sus márgenes. Si la usuaria ha cumplido su deseo reproductivo y es de difícil seguimiento o en etapa peri menopáusica se debe contemplarla posibilidad de histerectomía abdominal extra facial simple. 


\section{Materiales y métodos}

Es un estudio descriptivo cuali-cuantitativo de corte transversal, considerando el análisis de la información estadística de la Unidad de salud de atención a la mujer. También se desarrollaron entrevistas a pacientes que acudían a realizar chequeo ginecológico a esta unidad. El universo estuvo conformado por 38 mujeres diagnosticadas con lesiones pre malignas de cáncer cérvico uterino en el II semestre del año 2013, captadas en la unidad de atención primaria policlínico Ernesto Hodgson. Considerando que el universo es relativamente pequeño, se tomó como muestra el 100\% del universo.

Técnicas para la recopilación de información se consideraron como fuentes primarias las mujeres que fueron diagnosticadas con lesiones pre malignas de cáncer cervicouterino, con el fin de conocer su experiencia y percepción de la misma. También se consideraron los datos estadísticos y expedientes de la unidad de salud policlínico Ernesto Hodgson. Se utilizaron como técnicas: una guía de preguntas para las mujeres captadas en esta unidad de salud. También se realizó el llenado de una ficha clínica con datos obtenidos mediante la revisión del expediente clínico de las pacientes. El análisis y procesamiento de datos reflejados de estas investigaciones permitieron hacer comparaciones y análisis con los resultados para la profundización de explicaciones teóricas de este estudio. Lo cual contribuyo para la redacción del informe final.

Teniendo en cuenta el respeto a las mujeres que participaron voluntariamente, debido a que el tema de estudio se basa en una entidad relacionada al órgano reproductor femenino y por tratarse de temas de poco desarrollo social, no se ocupó el nombre de las mujeres durante llenado de instrumento, sino que se les asignó un código, el cual solo es del conocimiento de la autora principal del presente estudio tomando en cuenta la privacidad del paciente y la ética médica.

\section{Resultados y discusión}

\section{Perfil o características de mujeres afectadas con lesiones pre malignas de Cacu}

Edad: Sobre las edades de las mujeres consultadas, el 32\% son mujeres de 20-29 años, lo que demuestra que a pesar de mujeres multifactoriales se ha logrado disminuir el aumento de casos de mujeres con lesiones pre cancerosas avanzadas a temprana edad.

Seguido de las edades de 30-39 años que representan el 39\%, las mayores de 40 años que represen$\tan 18 \%$ que en relación con el tipo de lesión son las que se captaron con lesiones avanzadas es decir NIC III y un 11\% para mujeres de 15 a 19 años que en relación a los factores de riesgo en este caso de comportamiento sexual (IVSA a temprana a edad), se relaciona mayormente a atipias escamosas relacionadas con las infecciones por VPH y sus patologías asociadas como condilomatosis.

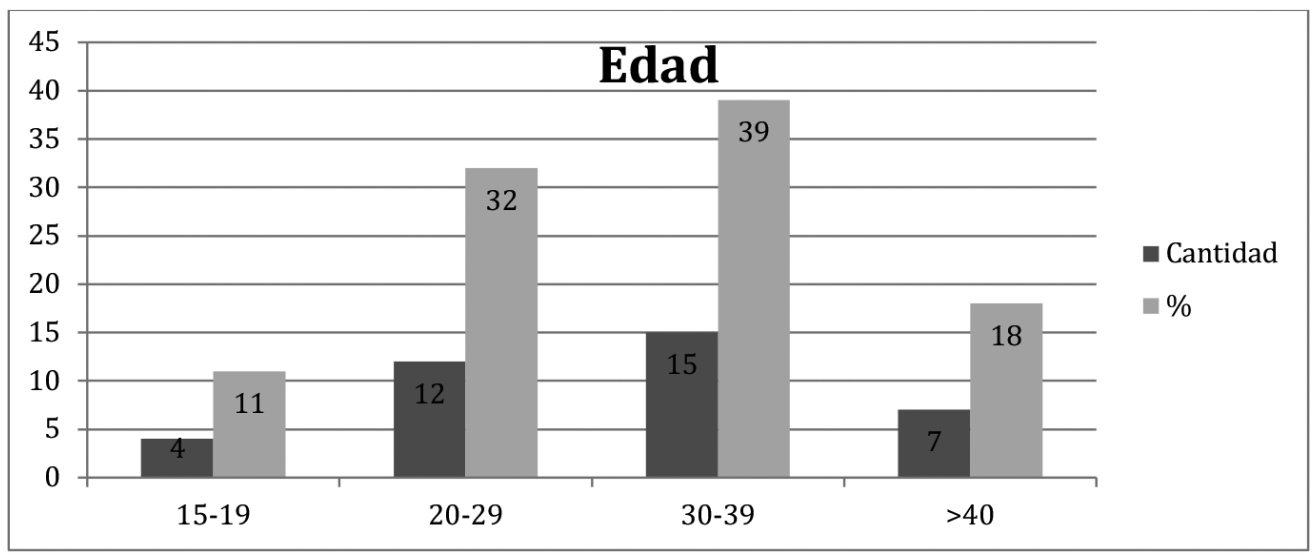

Figura 1: edad de pacientes con lesiones premalignas. 


\section{Edad de paciente}

Sobre las edades de las mujeres consultadas, el 32\% son mujeres de $20-29$ años, lo que demuestra que a pesar de mujeres multifactoriales se ha logrado disminuir el aumento de casos de mujeres con lesiones pre cancerosas avanzadas a temprana edad.

Seguido de las edades de 30-39 años que representan el 39\%, las mayores de 40 años que represen$\tan 18 \%$ que en relación con el tipo de lesión son las que se captaron con lesiones avanzadas es decir NIC III y un 11\% para mujeres de 15 a 19 años que en relación a los factores de riesgo en este caso de comportamiento sexual (IVSA a temprana a edad), se relaciona mayormente a atipias escamosas relacionadas con las infecciones por VPH y sus patologías asociadas como condilomatosis.

En relación a lo planteado por Serman (2002), se correlaciona ya que en este municipio se han captado mujeres con lesiones pre malignas que van desde 15 años a más, sin embargo, varían en relación al estadiaje por edad ya que algunas presentan más factores de riesgo en relación a otras, es decir no todas las mujeres tienen practicas inadecuadas o parejas sexuales de alto riesgo.

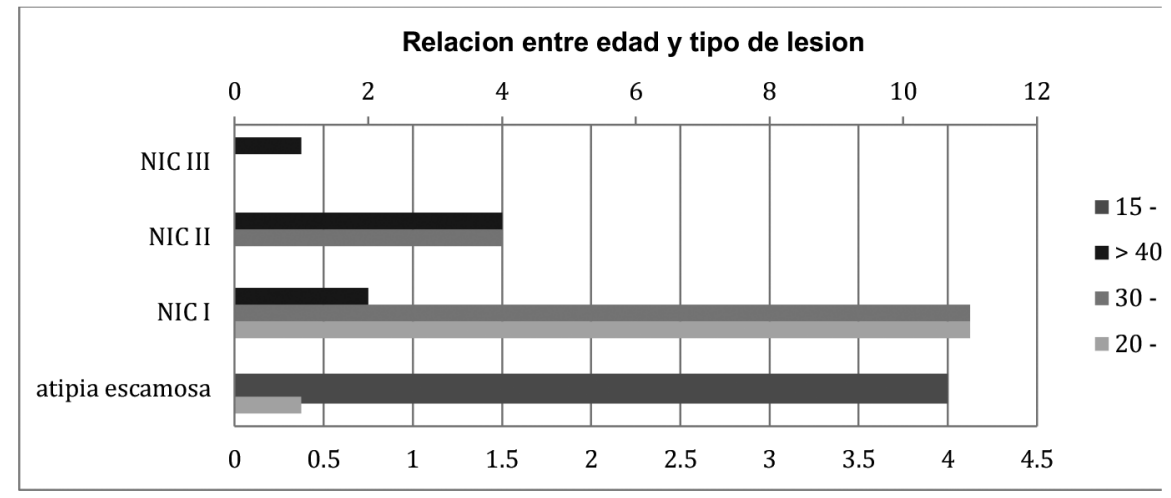

Figura 2: relación de edad y tipo de lesión.

\section{Edades y tipo de lesión}

1. Procedencia: $\mathrm{El} 74 \%$ es de la zona urbano asociado a la baja escolaridad que presentan las mujeres, la falta de prácticas de citologías como Papanicolaou y de información o conocimientos acerca del cáncer cervical y el nivel de urbanización presente en este municipio. En los datos de procedencia de las mujeres diagnosticadas con lesiones pre malignas o pre invasoras, en un $26 \%$ son de la zona rural esto asociado al nivel económico de las mujeres y la ausencia de servicios de salud en algunas de las comunidades.

2. Estado civil: Con respecto al estado civil la mayoría de las mujeres estudiadas mantenían uniones de hecho estables o acompañadas con un 42\%, esto representa a 16 mujeres. Un $34 \%$ equivalente a 13 mujeres son casadas y $21 \%$ que corresponde a 8 mujeres solteras.

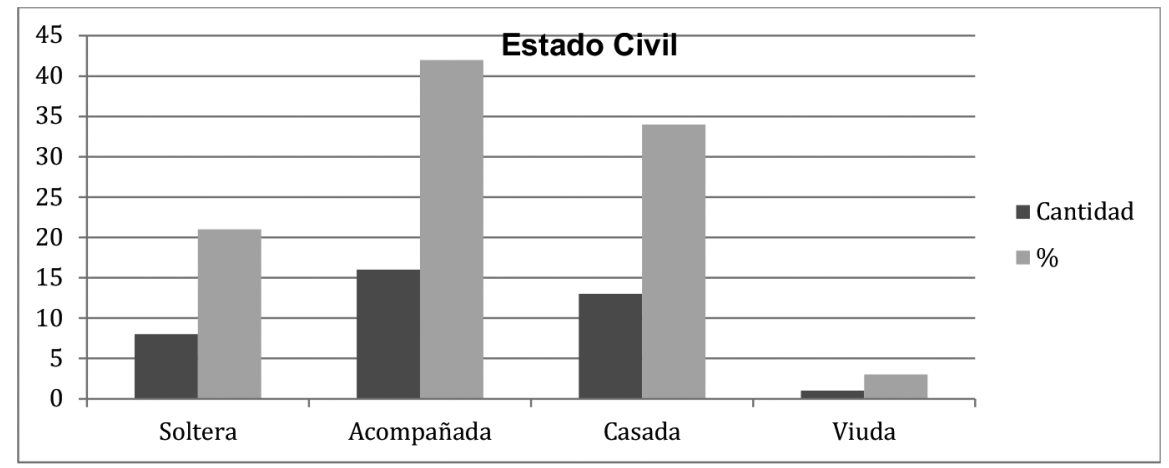

Figura 3: Estado civil de mujeres con lesiones premalignas. 


\section{Factores de riesgo}

Inicio de Vida Sexual Activa - IVSA: Según Gómez (2000) encontramos como factores de riesgos importantes para el desarrollo de lesiones pre invasoras, el Inicio de la Vida Sexual Activa a temprana edad antes de los 20 años y en otros casos antes de los 18. Esto es un cofactor para aparición tanto de embarazos a temprana edad, así como infecciones de transmisión sexual, lo que aumenta el riesgo de desarrollar lesiones pre cancerosas.

En las mujeres diagnosticadas con lesiones pre malignas en su mayoría el inicio de vida sexual activa lo realizaron en un 58\% entre los 14 a 16 años, un $26 \%$ tuvieron su primera experiencia sexual entre los 17 a 18 años. El 16\% igual o antes de los 13 años, edades de mucho riesgo y sobre todo de poca madurez física y emocional.

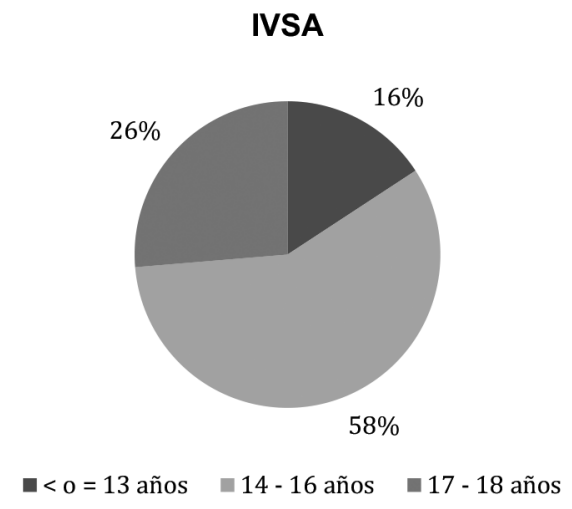

Figura 4. Inicio de vida sexual activa.

\section{IVSA}

Número de compañeros: En este factor de riesgo influye el estilo de vida sexual tanto de la mujer como de la pareja, con relación a la mujer se dice que a mayor cantidad de parejas mayor riesgo de desarrollar lesiones pre-malignas y más aún si las diferentes parejas han practicado la promiscuidad y sus anteriores parejas presentaban algún tipo de lesión o ITS, es decir la promiscuidad se considera una cadena de riesgos desconocidos.

\section{$\mathbf{N}^{\circ}$ de compañeros sexuales}

$\square N^{\circ}$ De compañeros sexuales

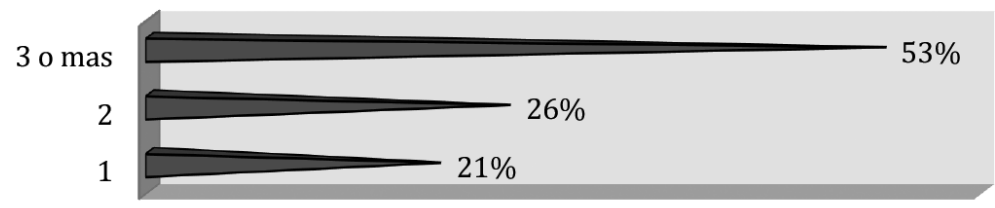

Figura 5: Numero de compañeros. 
En el análisis de la relación existente entre el número de parejas y el tipo de lesión se observó que las mujeres con mayor número de parejas sexuales es decir 3 o más (53\%), presentaron en su mayoría lesiones de leves a severas con NIC I 29\%, NIC II 21\% y NIC III (3\%) a excepción de las atipias escamosas lesiones de bajo grado, lo que hace énfasis en que la promiscuidad es un factor de gran importancia para el desarrollo de modificaciones en epitelio cervical, ya que se debe tener en cuenta que la mujer desconoce los antecedentes sexuales de cada pareja con la que sostuvo relaciones sexuales así como ( cantidad de parejas, antecedentes de ITS, parejas anteriores con VPH o algún tipo de lesión cervical, el uso de métodos de barrera e incluso higiene personal).

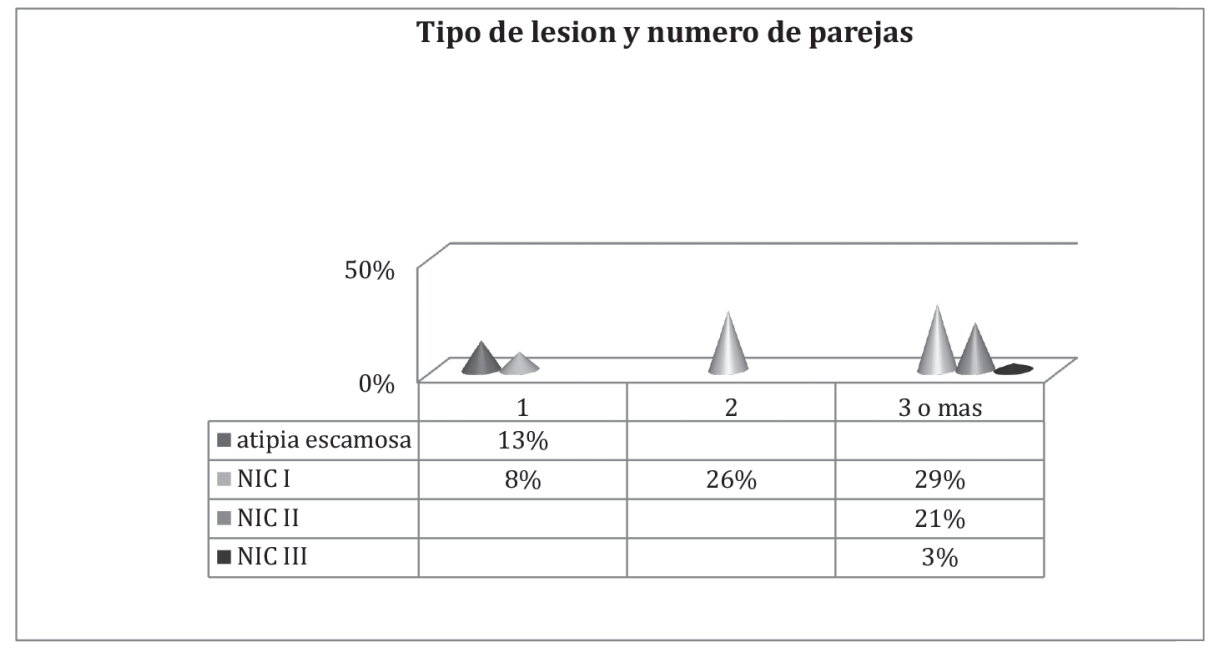

Figura 6: tipo de lesión y número de parejas.

\section{Relación entre tipo de lesión y numero de compañeros sexuales}

Número de hijos: durante el embarazo, parto y puerperio se dan muchos cambios tanto físicos como en los tejidos y órganos femeninos, en este caso durante el embarazo se dan una serie de cambios en el epitelio cervical en la llamada zona de transición lugar donde se inician las lesiones de riesgo y más aún cuando la mujer es portadora de ADN del VPH.

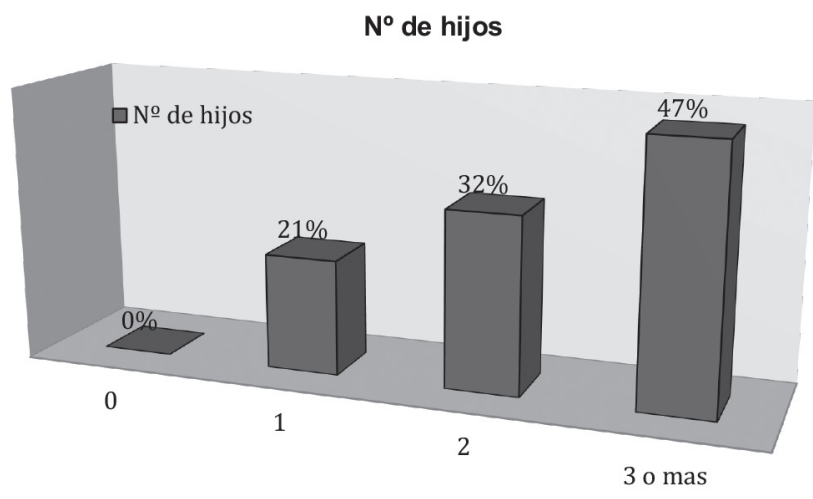

Figura 7: número de hijos.

Las mujeres de este estudio en su mayoría tuvieron 3 o más parejas sexuales lo que en relación con el tipo de lesión que desarrollaron todas estas presentaron lesiones como NIC I con un $24 \%$ y $23 \%$ NIC II. Un $3 \%$ presento NIC III que se correlaciona con factores de riesgo como procedencia rural, edad $>40$ años y estado civil viuda, todo esto desencadenan otros aspectos que influyeron tanto en la aparición de la lesión como en el seguimiento adecuado. 


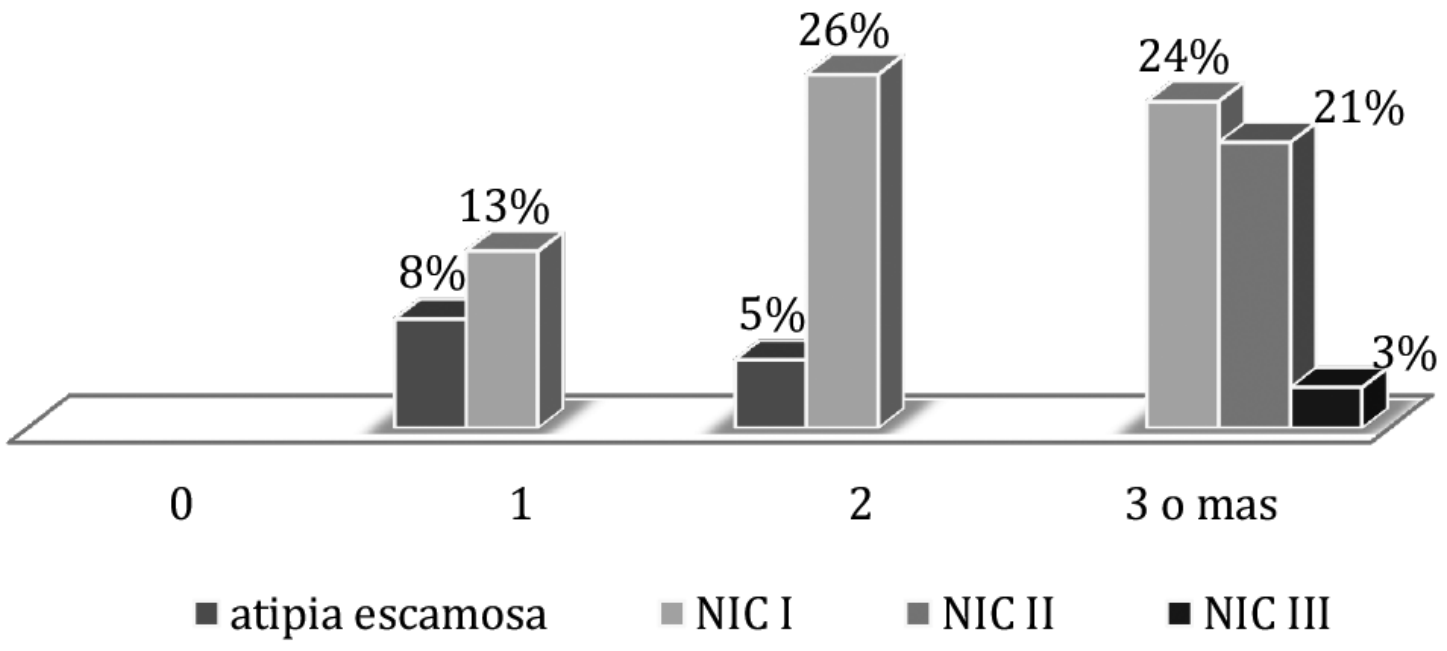

Figura 8. Relación entre número de hijos y tipo de lesión premaligna.

Lesiones premalignas: es un crecimiento anormal y pre-canceroso de células escamosas en el cuello uterino. La mayoría de los casos de NIC permanecen estables o son eliminados por el sistema del individuo sin intervención médica. Sin embargo un pequeño porcentaje de casos progresan a cáncer cervical, usualmente, por razón de la célula invasora, en un carcinoma de células escamosas.

\section{Lesiones Pre Malignas}

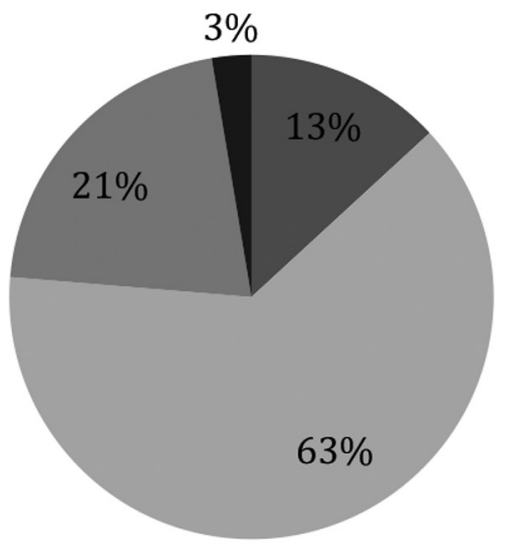

Atipias Escamo

NIC I

NIC II

NIC III

Figura 9: lesiones premalignas.

Según los datos obtenidos durante este estudio de los 38 casos de mujeres con lesiones premalignas en un $63 \%$ las mujeres se captaron en lesión leve que corresponde al NIC I, seguido de lesión moderada NIC II con un 21\%, y en lesión severa NIC III un 3\% afortunadamente la minoría. Sin embargo, un 13\% fueron captadas como atipia escamosa en las cuales se incluyen las infecciones por VPH.

\section{Relación de preferencia de manejo y procedencia}

La RACCN se caracteriza por ser una región multiétnica y pluricultural, donde los pueblos indígenas y por tanto las mujeres tienen una percepción del bienestar físico es decir del proceso salud - enfermedad muy diferente a otras regiones del país, en nuestra región el equilibrio entre la naturaleza y lo pobladores de los diferentes territorios juega un papel muy importante en este proceso. 
Así mismo cuando no se contaba con los servicios ni suministros adecuados de personal de salud y medicinas, era el médico tradicional, partera o Sukia quien sanaba o curaba todas y cada una de las dolencias de la población, y aunque los años han pasado estos siguen teniendo igual importancia para la población es por lo que se creó un modelo de salud culturalmente apropiado.

En el entorno de las enfermedades específicamente de las mujeres la partera es la que desempeña un papel muy importante ya que por su sabiduría y experiencia de años las mujeres confían en sus habilidades y conocimientos y prefieren ser tratadas por ellas.

\section{Preferencia de manejo según procedencia}

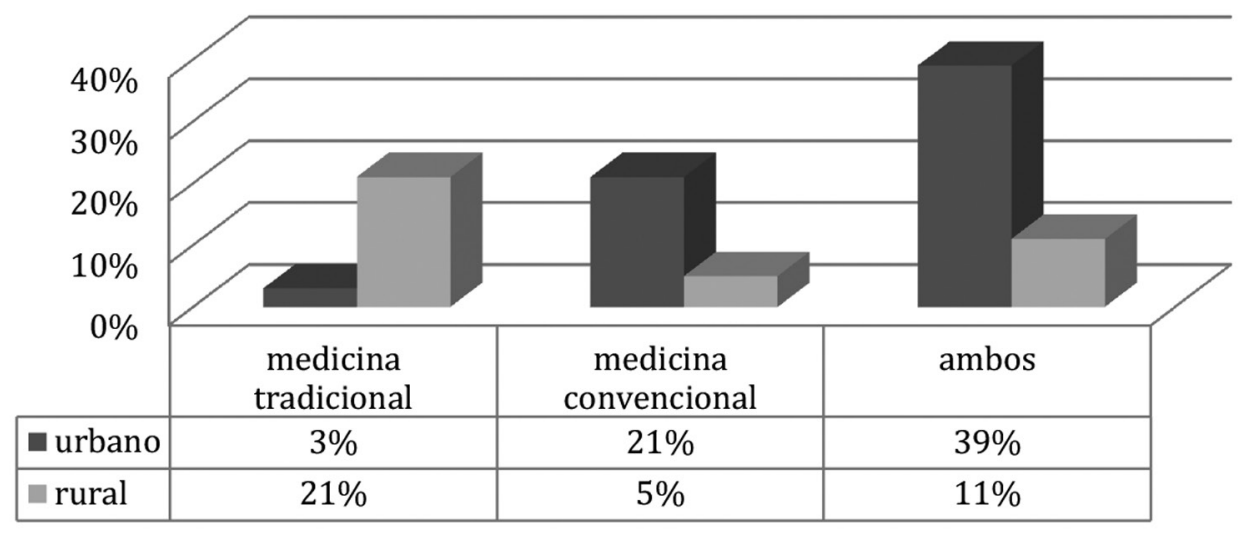

Figura 10: preferencia de manejo por procedencia.

La mayoría de las mujeres de procedencia urbana que participaron en el estudio tenían la percepción de ser tratadas desde un ambiente donde se implemente la medicina convencional y tradicional en un 39\%, ya que de esta manera tenían mayor aprobación y seguridad acerca del manejo.

Sin embargo, un $21 \%$ tanto rural como urbano se encuentran en que los de procedencia rural tienen tendencia a preferir ser tratadas tradicionalmente debido a la relación de confianza y seguridad que conservan con sus médicos tradicionales y parteras. El mismo porcentaje, pero de procedencia urbana opta por ser tratadas convencionalmente ya que refieren que hay problemas de salud que deben ser tratados de la forma medica convencional, aun así, conservan su cosmovisión del proceso salud enfermedad.

Manejo: A nivel nacional el ministerio de salud ha realizado normativas con el objetivo de establecer medidas de prevención, detección y manejo de lesiones premalignas de cáncer cérvicouterino, así como del cáncer invasor. Este manejo es exclusivamente a través de la medicina occidental ya sea dando seguimiento con controles de Papanicolaou, realización de colposcopia o crioterapia en algunos casos como biopsias la cual, en un método de diagnóstico, pero también tratamiento.

Sin embargo, en estas normativas desde la perspectiva de las mujeres no se adapta a las necesidades de la mujer costeña, ya que no se incorpora el aspecto intercultural, es decir no se toma en cuenta que dicha patología desde su cosmovisión no es solamente un problema médico, sino también un problema que se relaciona con entidades culturales las cuales solamente son tratadas por los médicos tradicionales o parteras, incluso influye el aspecto religioso. 


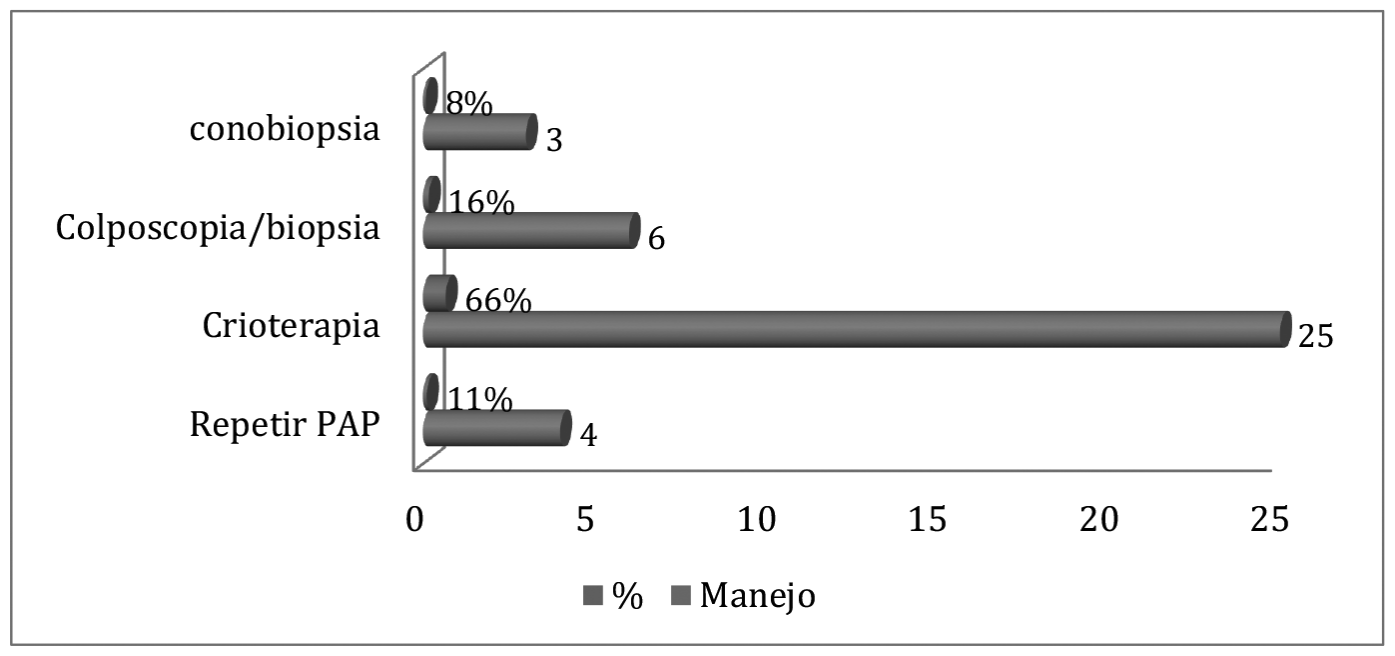

Figura 11: manejo de lesiones pre malignas.

Las lesiones pre malignas así como puede revertirse espontáneamente a través del sistema inmune, así mismo pueden desarrollarse, es por eso que en su mayoría se les dio seguimiento a través de colposcopia medio por el cual a la vez se puede realizar biopsias $16 \%$ y a veces se mantiene manejo expectante es decir en un $11 \%$ a las mujeres se les envió a repetir el PAP, sin embargo en su mayoría se les realizo crioterapia en un 66\% un método poco invasivo, en su minoría se les realizo cono biopsias este procedimiento tiene dos acciones es un método diagnostico pero también es tratamiento en lesiones pre malignas severas es decir NIC III, un $8 \%$ de las mujeres fueron sometidas a este procedimiento.

\section{Conclusiones}

El cáncer cervical es uno de los tipos de canceres que más afecta a las mujeres costeñas este ocupa el primer lugar en Puerto Cabezas. Sin embargo, se han estado captando un gran porcentaje de mujeres con $\mathrm{PAP}$ en lesiones premalignas en su mayoría con lesiones iníciales las cuales se pueden manejar a tiempo.

Según el estudio realizado, las mujeres de nuestro municipio tienen los factores de riesgo descritos, tales como el inicio de vida sexual en edad temprana, paridad temprana, múltiples compañeros sexuales, parejas promiscuas entre otros como la presencia de infecciones de transmisión sexual (ITS) y el Virus del Papiloma Humano (VPH), lo que nos indica que son mujeres multifactoriales de alto riesgo.

También se encontró que de todos los factores de riesgo que predisponen a desarrollar lesiones los de mayor frecuencia en las mujeres de este municipio son los de tipo sexual es decir: múltiples compañeros sexuales, inicio de vida sexual activa a temprana edad y por ende paridad a temprana edad, y en su mayoría la detección y seguimiento se debió a la falta de conocimientos y actitud de responsabilidad de las mujeres acerca de la importancia de los chequeos y exámenes ginecológico con frecuencia.

Otro aspecto importante encontrado es que en la mayoría de las mujeres que fueron diagnosticadas con lesiones premalignas de cáncer cervical no fue posible un adecuado seguimiento ya que en la mayoría son de pocos recursos económicos y baja escolaridad con pocos conocimientos acerca de la importancia de seguimiento y realización de PAP.

Además, que muchas desde su cultura y cosmovisión el manejo de este problema de salud debería ser promocionado en conjunto con sus médicos tradicionales por los aspectos culturales que las identifican. Es importante destacar que la mayoría de las pacientes que fueron diagnosticadas con lesiones 
premalignas de cáncer cervical se encontraron en unas etapas iniciales como es un NIC I Y NIC II algunos casos se encontraron en NIC III, por lo que el manejo brindado a través de seguimiento fue la realización crioterapia en etapas iníciales y en casos avanzados cono biopsias.

En la mayoría de los casos el diagnostico se realizó al cabo de 3 meses posteriores a la realización del tamizaje y el tratamiento en un mínimo de 2 meses posteriores del diagnóstico en dependencia de la etapa en caso de los NIC II, en su mayoría referidas al Hospital "Nuevo Amanecer" para posterior seguimiento desde el centro de salud donde fueron captadas.

Por lo tanto es de gran importancia implementar la realización de medios diagnósticos para el cáncer cervical en mujeres en edad reproductiva con vida sexual activa en este caso el Papanicolaou, que está más al alcance de las mujeres en nuestra región así como fortalecer más los programas educativos sobre los factores de riesgos implicados en el desarrollo de lesiones pre malignas la educación de la conducta sexual y reproductiva que incluye métodos de protección y por ende de planificación familiar.

Sobre todo, programas con enfoques interculturales que integren a los médicos tradicionales y parteras para que de esta manera se logre sensibilizar a las mujeres del auto cuidado y la importancia de un diagnóstico temprano, y de la importancia de la asistencia a chequeos ginecológicos, con el objetivo de disminuir el riesgo de desarrollar lesiones pre malignas y mejorar el estado de salud y condición de vida de cada una de las mujeres de los pueblos indígenas de esta región. Además de la educación continua con información básica e intercambio de conocimientos con los cita y médicos tradicionales entidades reconocidas con la mayor sabiduría de las comunidades más alejadas para que de esta forma se promueva el modelo salud adaptado a la región MASI RAAN para que de esta manera se disminuyan las brechas y se entable una mejor relación para la práctica de la verdadera medicina intercultural a favor de la población femenina de esta región.

\section{Lista de referencias}

Aguilera, A. (2008). Cáncer cervicouterino provocando estragos. Managua, Nicaragua. El Nuevo Diario, 3b.

Schwarz, R. (2008). Obstetricia. 6ta edición, Buenos Aires.

Serman, F. (2002). Cáncer cervicouterino: epidemiologia, historia natural y rol del virus papiloma humano. Revista chilena de obstetricia y ginecología, v.67 n4, 318-323.

MINSA (2010), Normativa 037 técnica de prevención, detección y atención del cáncer cervicouterino. MINSA, SILAIS. Managua, Nicaragua.

Organización Mundial de la Salud (OMS, 2000. Control integral del cáncer cérvico uterino. Guía de prácticas esenciales. México.

Organización Panamericana de la Salud (OPS, 2002). Planificación de programas apropiados para la prevención del cáncer cérvico uterino zra edición. 